\title{
Role of yttrium-90 selective internal radiation therapy in the treatment of liver-dominant metastatic colorectal cancer: an evidence-based expert consensus algorithm
}

\author{
D. Rohan Jeyarajah ${ }^{1}$, Maria B. Majella Doyle ${ }^{2}$, N. Joseph Espat ${ }^{3}$, Paul D. Hansen ${ }^{4}$, David A. Iannitti ${ }^{5}$, \\ Joseph Kim ${ }^{6}$, Thavam Thambi-Pillai ${ }^{7}$, Brendan C. Visser ${ }^{8}$ \\ ${ }^{1}$ Methodist Richardson Medical Center, Richardson, TX, USA; ${ }^{2}$ Washington University School of Medicine in St. Louis, St. Louis, MI, USA; \\ ${ }^{3}$ Department of Surgery, Roger Williams Medical Center, Boston University School of Medicine, Providence, RI, USA; ${ }^{4}$ HPB Surgery, Providence \\ Portland Center, Portland, OR, USA; ${ }^{5}$ HPB Surgery, Atrium Health, Carolinas Medical Center, Charlotte, NC, USA; ${ }^{6}$ Department of Surgery, \\ University of Kentucky, Lexington, KY, USA; ${ }^{7}$ Department of Surgery, University of South Dakota Sanford School of Medicine, Sioux Falls, SD, \\ USA; ${ }^{8}$ Department of Surgery, Stanford University Medical Center, CA, USA \\ Contributions: (I) Conception and design: All authors; (II) Administrative support: None; (III) Provision of study materials or patients: All \\ authors; (IV) Collection and assembly of data: All authors; (V) Data analysis and interpretation: All authors; (VI) Manuscript writing: All authors; \\ (VII) Final approval of manuscript: All authors. \\ Correspondence to: D. Rohan Jeyarajah, MD. Methodist Richardson Medical Center, 2805 E. President George Bush Highway, RichardsonM, TX \\ 75082, USA. Email: rohanjeyarajah@gmail.com.
}

\begin{abstract}
Surgical resection of colorectal liver metastases is associated with greater survival compared with non-surgical treatment, and a meaningful possibility of cure. However, the majority of patients are not eligible for resection and may require other non-surgical interventions, such as liver-directed therapies, to be converted to surgical eligibility. Given the number of available therapies, a general framework is needed that outlines the specific roles of chemotherapy, surgery, and locoregional treatments [including selective internal radiation therapy (SIRT) with Y-90 microspheres]. Using a data-driven, modified Delphi process, an expert panel of surgical oncologists, transplant surgeons, and hepatopancreatobiliary (HPB) surgeons convened to create a comprehensive, evidence-based treatment algorithm that includes appropriate treatment options for patients stratified by their eligibility for surgical treatment. The group coined a novel, more inclusive phrase for targeted locoregional tumor treatment (a blanket term for resection, ablation, and other emerging locoregional treatments): local parenchymal tumor destruction therapy. The expert panel proposed new nomenclature for 3 distinct disease categories of liver-dominant metastatic colorectal cancer that is consistent with other tumor types: (I) surgically treatable (resectable); (II) surgically untreatable (borderline resectable); (III) advanced surgically untreatable (unresectable) disease. Patients may present at any point in the algorithm and move between categories depending on their response to therapy. The broad intent of therapy is to transition patients toward individualized treatments where possible, given the survival advantage that resection offers in the context of a comprehensive treatment plan. This article reviews what is known about the role of SIRT with Y-90 as neoadjuvant, definitive, or palliative therapy in these different clinical situations and provides insight into when treatment with SIRT with Y-90 may be appropriate and useful, organized into distinct treatment algorithm steps.
\end{abstract}

Keywords: Metastatic colorectal cancer; selective internal radiation therapy (SIRT); yttrium-90; local parenchymal tumor destruction therapy; transarterial radioembolization

Submitted Sep 20, 2019. Accepted for publication Jan 10, 2020.

doi: 10.21037/jgo.2020.01.09

View this article at: http://dx.doi.org/10.21037/jgo.2020.01.09

(C) Journal of Gastrointestinal Oncology. All rights reserved. 


\section{Introduction}

The liver is a common site of metastatic tumor development in patients with advanced colorectal cancer (CRC) (1); liver metastases are present in approximately $20-35 \%$ of patients with CRC at diagnosis and in up to $70 \%$ at death (1-3). The median survival of patients with untreated CRC liver metastases (CRCLM) after diagnosis is 6-12 months, with survival after 5 years being rare (4). Surgical resection of CRCLM has shown 5-year survival rates of approximately $20-70 \%$ and should be the goal for eligible patients (5). Unfortunately, the majority (70-80\%) of patients with liverdominant disease are not suitable candidates for resection due to technical reasons, severe comorbidities, or significant extrahepatic disease $(1,5,6)$. The goal of current medical treatment for patients with borderline resectable CRCLM is to improve tumor response rates to maximize the potential for curative resection (7). However, for patients with chemorefractory CRCLM, there is no standard recommended treatment course (8).

\section{Identifying patients with liver mCRC for appropriate treatment}

Decisions relating to patient selection for specific treatment choices are critical in the management of mCRC (5) and include decisions about the type and duration of treatment (9). Treatment goals for patients with mCRC have been classified as curative intent ("resectable" or "operable"), potentially curative, non-curative with active treatment intent, and non-curative with palliative intent (i.e., best supportive care) $(10,11)$. Choice of first-line therapy initiates the treatment sequence, leading to subsequent treatment decisions for next-line therapies, which depend on both the treatment goal and the molecular subtype of the tumor (11). The sequence of treatments is also important given the potential for recurrence requiring subsequent treatment with additional lines of therapy and the potential interaction between them (11).

To confound these decisions, the definition of resectability seems to differ in clinical practice, and patients with liver-dominant $\mathrm{mCRC}$ who are classified as having unresectable disease in one institution might be said to have resectable disease in another institution $(12,13)$. Even among experienced surgeons, there is variability in decision-making, where some choose to resect while others are more conservative (7). When resectability of CRCLMs were examined by both colorectal and liver specialist teams, management decisions were divergent in almost half of patients (13). Thus, many patients with potentially resectable CRCLM are not being considered for potentially beneficial treatment by appropriate specialists (13). Patient evaluation by a clinically experienced liver surgeon is essential to ensure that resection is appropriately considered in each patient's multidisciplinary treatment strategy $(4,7,14)$. To provide a potentially curative option for patients with liver-limited, unresectable mCRC that cannot be resected until regression of tumors from critical structures, conversion strategies can be used to downsize and convert CRCLM to a resectable status $(5,7)$.

\section{Current treatment options for CRCLM}

Systemic chemotherapy has been shown to yield high tumor response rates that result in appreciable rates of conversion to resectability, including irinotecan-based or oxaliplatin-based regimens in combination with antiepidermal growth factor receptor (EGFR) inhibitors such as cetuximab, with rates being reported between $4 \%$ and $60 \%$ for downsizing with chemotherapy alone $(5,7,15-17)$. Posttreatment resectability has been associated with better overall survival (OS), which demonstrates the importance of conversion chemotherapy (12). In patients who are converted to resection with chemotherapy, 5-year survival can approach $30-50 \%$, with $20 \%$ of patients alive 10 years after resection (17). Intra-arterial therapies may also be used to downsize metastases to allow resection, even in patients who have disease refractory to chemotherapy (18). New locoregional liver-directed therapies are emerging but have not yet become the standard of care in many practices (6). As metastatic tumors greater than $3 \mathrm{~mm}$ are supplied primarily by the hepatic artery (19), treatments administered via the hepatic arterial system enhance delivery of tumoricidal and embolizing agents to tumor tissue and may reduce significant hepatic and systemic toxicity $(8,18,20)$. Such treatments include hepatic arterial infusion pump (HAIP) therapy, conventional transarterial chemoembolization (cTACE), drug-eluting bead transarterial chemoembolization (DEB-TACE), and selective internal radiation therapy (SIRT) with yttrium-90 (Y-90) [also called intra-arterial brachytherapy, transarterial radioembolization (TARE)] $(8,21,22)$. With the diversity of available systemic and locoregional treatments for patients with $\mathrm{mCRC}$, establishing an effective management approach may be challenging, in part due to a lack of comparative studies and differences in institutional practices $(8,23)$. 
SIRT is an arterially delivered brachytherapy that percutaneously delivers microspheres containing Y-90, a beta-emitting radionuclide, directly into the tumor via the hepatic artery (6). SIRT with Y-90 has been found to cause tumor necrosis and fibrosis and has effects on dystrophic calcifications and tumor vasculature (24). Evidence suggests that SIRT with Y-90 may improve treatment response in patients with chemorefractory mCRC (25) and in patients receiving 5 -fluorouracil (5-FU) chemotherapy (26), and may also delay liver disease progression when administered as first-line therapy with chemotherapy (16). Furthermore, SIRT has been used as a tool to downsize select patients with CRCLM with increases in resectability (27) and actual resection rates that have ranged between $10 \%$ and $21 \%$ $(15,16,28,29)$. However, most patients who receive SIRT with Y-90 present in the salvage setting, with extensive liver tumor load, reduced performance status, chemorefractory disease, and many prior chemotherapy cycles (30). In fact, these heavily treated patients are poor candidates for any type of therapy, often having disease that failed to respond to all other evidence-based treatments (31). Outside of broad guidelines provided by the US National Comprehensive Cancer Network (NCCN) and European Society for Medical Oncology (ESMO), the need for consensus remains for the specific roles of SIRT with Y-90 for patients with CRCLM (32-34).

\section{Need for evidence-based consensus for treatment}

Arterially directed catheter therapy and, in particular, SIRT with Y-90 microspheres, has been included in the ESMO and NCCN guidelines for the management of patients with mCRC $(5,35)$. The NCCN guidelines recommend SIRT with Y-90 for treatment of mCRC in highly selected patients with chemotherapy-resistant/-refractory disease and with predominant hepatic metastases (Category 2A recommendation) (5) but requires further evidence to recommend a role for this treatment at earlier stages of mCRC (5). The ESMO guidelines recommend SIRT with Y-90 for patients with liver-limited disease that has failed the available chemotherapeutic options (Level II evidence, Grade B recommendation) and leaves room for potential earlier use as "consolidation treatment" (35).

Despite the inclusion of intra-arterial therapies in these guidelines, consensus is needed among oncology specialists regarding the exact sequence involved in treating different stages of liver-dominant mCRC, particularly the specific roles of chemotherapy, surgery, and locoregional treatments
$(1,33)$. There is need for evidence-based, multidisciplinary consensus with respect to the specific role of SIRT with Y-90 in the treatment of patients with varying degrees of liverdominant mCRC (32) as well as criteria for appropriate patient selection (36).

To this end, a multi-step series of expert panels was convened with experienced practitioners, including surgical oncologists, transplant surgeons, and hepatopancreatobiliary (HPB) surgeons, to review current clinical evidence on the role of SIRT with Y-90 in the treatment landscape of mCRC. The result was a data-driven treatment algorithm based on the surgical resectability/tumor destruction status of patients, with the goal of moving patients toward eligibility for curative or disease-stabilizing therapy. The expert panels sought to investigate practical considerations, grade the evidence, and provide guidance on key considerations in the management of different clinical situations for patients with liver-dominant mCRC. This paper describes the development process of the proposed treatment algorithms, which included a systematic literature review followed by successive modified Delphi-based expert panels. The evidence-supported treatment algorithms focus on key considerations of mCRC treatment regarding the role of SIRT with Y-90.

\section{Methods}

\section{Study design}

This evidence-based treatment algorithm was developed by a multidisciplinary expert panel of surgical oncologists, transplant surgeons, and HPB surgeons with extensive experience in treating mCRC. To develop the algorithm, a multi-step modified Delphi process was conducted, which provides a structured method of group communication to facilitate the aggregation of expert opinions and reach an agreement on complex issues (37). This process involved multiple stages between October 2018 and March 2019 (Figure 1). First, a Core Expert Panel $(\mathrm{N}=8)$ met at 2 live meetings, as well as several webinars, and corresponded through email.

The Core Expert Panel had 2 main goals. Firstly, it aimed to define distinct disease categories for patients with liver-dominant mCRC stratified by surgical treatability. Secondly, it aimed to create a treatment algorithm that detailed evidence-supported recommendations on appropriate treatment options for patients at various degrees of eligibility for surgical treatment, with an emphasis on 


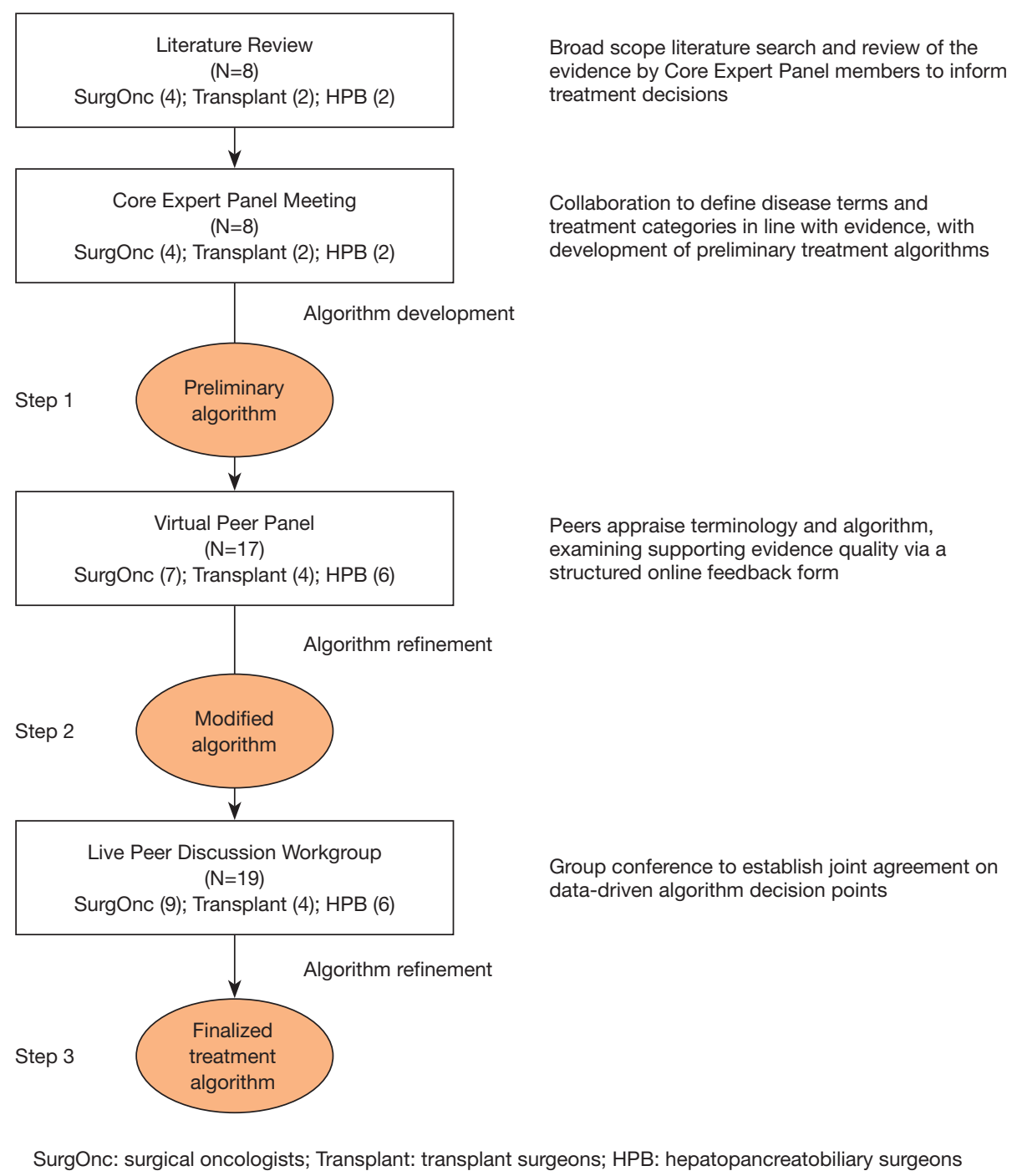

Broad scope literature search and review of the evidence by Core Expert Panel members to inform treatment decisions

Collaboration to define disease terms and treatment categories in line with evidence, with development of preliminary treatment algorithms

Peers appraise terminology and algorithm, examining supporting evidence quality via a tructured online feedback form

Group conference to establish joint agreement on data-driven algorithm decision points

Figure 1 Multi-step modified Delphi process.

when SIRT with Y-90 would be appropriate.

A blinded, broad-scope literature review was conducted to find publications on the role of SIRT with Y-90 in patients with mCRC (Figure 2). Eligible studies included systematic reviews and meta-analyses, randomized controlled trials (RCTs), phase I and II trials, prospective and retrospective cohort studies, case series, and consensus guidelines. Excluded publication types included nonsystematic reviews, single case reports, those involving only non-CRCs or primary liver tumors, and studies published before 2009. Levels of evidence were assigned based on study type according to criteria from the Oxford Centre for Evidence-Based Medicine (38). Articles were selected based on eligibly criteria and content relevant to the review.
In addition to any published data analyzing the role of SIRT with Y-90 in mCRC, several specific themes were of interest because they were relevant to the specific intent to use SIRT with Y-90 to help move patients to surgical therapy. These included studies that discussed the role of SIRT with Y-90 in converting borderline resectable tumors to resectable status, inducing contralateral hypertrophy, treating small metastases completely, and treating disease while testing tumor biology with chemotherapy. After this review, 30 papers were deemed eligible and relevant and were included. Core Expert Panel members were given the primary literature to perform a detailed review of the evidence.

Based on the evidence, the Core Expert Panel developed 


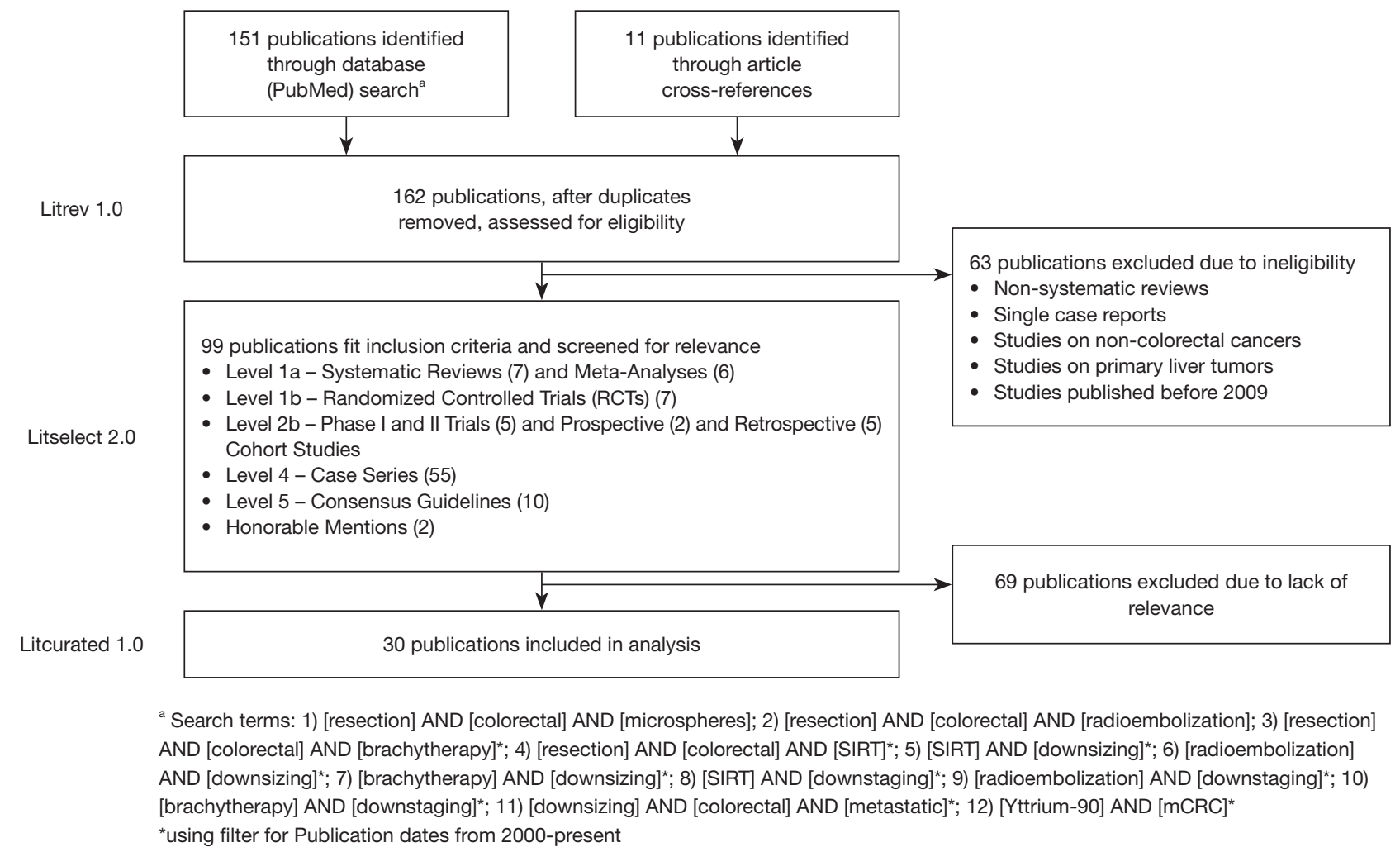

Figure 2 Literature review on the role of selective internal radiation therapy (SIRT) with Y-90 in patients with metastatic colorectal cancer (mCRC).

the preliminary algorithm (Step 1), provided critical review, and finalized the algorithm structure. The Core Expert Panel reviewed and approved the preliminary version of the algorithm. Ratings for the strength of evidence were provided with each treatment step.

The algorithm and supporting evidence levels were then scrutinized by a larger Virtual Peer Panel $(\mathrm{N}=17)$ to create a modified algorithm (Step 2) in which key issues with decision points were identified. Based on the peer review, revisions were made to the draft to clarify treatment steps for clinical practice. A second in-person discussion workgroup meeting with a Live Peer Discussion Workgroup ( $\mathrm{N}=19)$ was conducted to discuss these issues and further refine the algorithm. These treatment steps were refined and modified until agreement was reached to create a finalized algorithm (Step 3). If no consensus was achieved, the alternative views of the group were noted and are described herein. The group focused on creating a cohesive map for treating patients with $\mathrm{mCRC}$, with an emphasis on the algorithm steps and their relative order. Attention was placed on arriving at a consensus about the overarching algorithm instead of explicitly measuring the degree of agreement on each individual step. Many individual treatment steps within the algorithm were based on already-established recommendations from NCCN and ESMO guidelines, referenced where appropriate. Evidence supporting all individual steps was added, including the levels of evidence of each study.

\section{Results}

\section{Terminology for locoregional treatments}

During discussion of terminology, the Core Expert Panel found that the terms ablation and resection excluded the use of other locoregional treatments, and determined a need for a more concise term to refer to tumor ablation or destruction technologies that are intended for complete local control that would otherwise be equivalent to resection (39-41). A more inclusive term for targeted locoregional tumor treatment, local parenchymal tumor destruction therapy, is a blanket term for both resection and ablation as well as other emerging locoregional treatments. This term leaves 


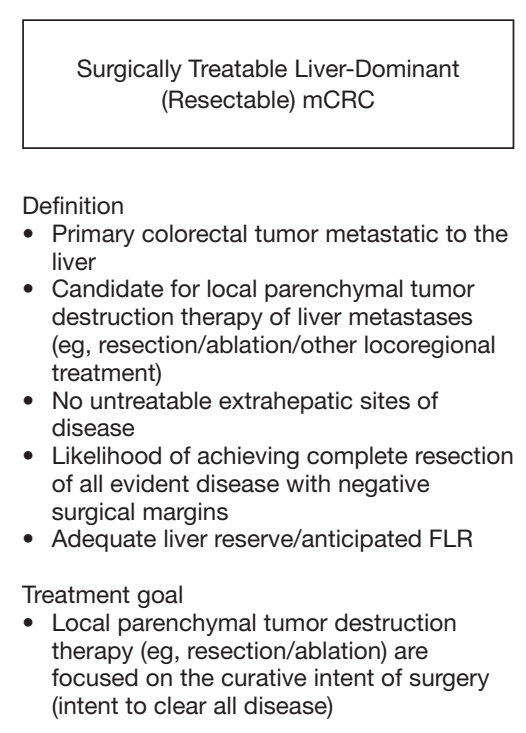

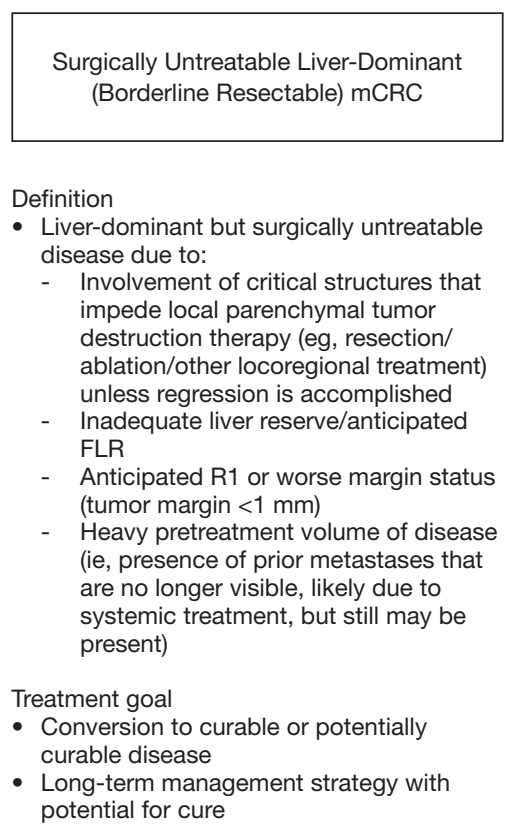

Advanced Surgically Untreatable Liver-Dominant (Unresectable) mCRC

Definition

- Not amenable to local parenchymal tumor destruction therapy of liver metastases (eg, resection/ablation)

Treatment goal

- Focus on disease palliation

Figure 3 Treatment categories. The Core Expert Panel developed 3 categories of patients with liver-dominant metastatic colorectal cancer (mCRC) stratified by surgical treatability. FLR, future liver remnant.

room for the integration of future novel tumor therapies into the therapeutic armamentarium for patients with mCRC.

\section{Treatment categories}

When considering treatment approaches for different patient types, treatment goals are different depending on the status of the patient. The Core Expert Panel identified 3 main disease categories for patients with liver-dominant mCRC stratified by surgical treatability and stipulated the general treatment goals for each group (Figure 3):

(I) Surgically treatable liver-dominant (resectable) mCRC: patients who are eligible for surgical treatment (curative intent);

(II) Surgically untreatable liver-dominant (borderline resectable) mCRC: patients who may be eligible for surgical treatment but are currently untreatable due to oncologic or technical factors and could be potentially converted to resectable status;

(III) Advanced surgically untreatable liver-dominant (unresectable) mCRC: patients who are not amenable to surgical treatment at the time of entry into the algorithm.

Of note, the use of this terminology for categories of surgical treatability mirrors treatment categories used in pancreatic cancer classification (42), for which the goal is to identify patients who would benefit from surgery by dividing patients into 3 similar treatment categories (42). They also echo the general views across the field of mCRC treatment, where it is understood that patients with CRCLM can generally be classified into resectable, potentially resectable/ convertible, and unresectable groups $(5,23)$. Broad definitions of each disease category are outlined below and are meant to be used as rough parameters to choose the most appropriate treatment. When evaluating individual patients for treatment, physicians should consider not only disease-related factors such as extent of intrahepatic disease, presence/extent of extrahepatic disease, timing of metastatic diagnosis (synchronous versus metachronous), and disease biology, but also patient-related variables such as age, comorbidities, preferences, and expectations (23).

Based on the categories of disease, the Core Expert Panel created a distinct treatment algorithm for each group, taking into account the unique considerations associated with each stage of disease. Each algorithm is a distinct entity. An overarching framework of the treatment algorithm is provided in Figure 4. Patients can enter the algorithm at any point and move vertically or laterally, depending on treatment response. The overarching goal 


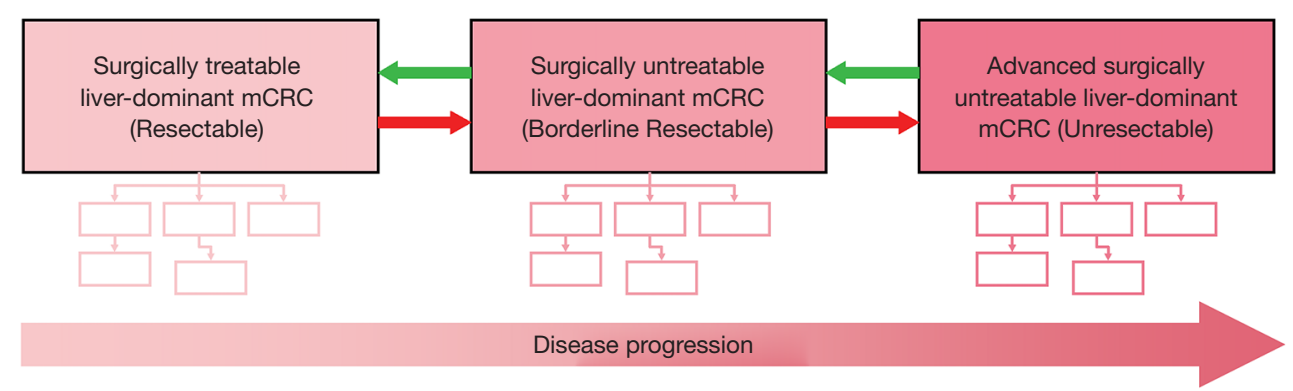

Figure 4 Treatment algorithm framework. The Core Expert Panel developed a distinct treatment algorithm for each group based on surgical treatability. Patients enter at any point and move vertically or laterally depending on response to treatment. mCRC, metastatic colorectal cancer.

of treatment is to transition patients toward eligibility for curative interventions or to achieve prolonged disease-free intervals.

\section{Category 1: Surgically treatable liver-dominant (resectable) mCRC}

Patients with surgically treatable liver-dominant (resectable) mCRC have a primary colorectal tumor metastatic to the liver $(5,35)$, no untreatable extrahepatic sites of disease (5), and adequate liver reserve $(5,35)$. They must be candidates for local parenchymal tumor destruction therapy of liver metastases (5). In these patients, there is a likelihood of achieving complete resection of all evident disease with negative surgical margins $(5,35)$. The goal of treatment is to clear all disease, whether through local parenchymal destruction therapy or other methods $(5,35)$.

The treatment of patients with surgically treatable liverdominant (resectable) mCRC is outlined in Figure $5(5,35)$. Patients with minimal disease (oligometastases) may be, in some situations, appropriate candidates for immediate surgical treatment, whereas patients with more extensive disease (metachronous, poorly differentiated) may require neoadjuvant or adjuvant chemotherapy to test disease trajectory and/or downsize lesions before local parenchymal tumor destruction therapy $(5,35)$. Patients with a resectable primary colon tumor and resectable synchronous metastases can be treated with a synchronous or staged resection of the liver with curative intent (or a combination of liver resection and/or local tumor destruction, where equivalent, for CRCLM) $(5,35,43)$.

However, not all patients with technically resectable liver-limited metastases will ultimately benefit from surgery, with approximately half developing widespread systemic disease within 3 years after resection (35). For some patients, neoadjuvant chemotherapy may be a better option than immediate surgery (35). An initial course (4-6 cycles) of systemic first-line chemotherapy is also a reasonable strategy for use in patients with resectable mCRC at high risk for recurrent disease, although evidence to support this approach is somewhat mixed $(5,18,35)$. The expert panelists concurred that a neoadjuvant approach merits strong consideration in patients with any unfavorable factor, e.g., an extensive burden of liver disease, a short interval from primary diagnosis to the appearance of metastases, or extrahepatic disease $(33,35)$. Response to chemotherapy is used by many investigators as a surrogate marker of tumor biologic behavior and may be used to select for hepatectomy in some patients (18). In fact, pathologic response to chemotherapy has been an important prognostic factor in patients who undergo resection for metastatic CRC (44). Radiographic assessment by CT and cross-sectional imaging may be performed often to assess for extrahepatic metastases and local recurrence $(5,35)$. Magnetic resonance imaging (MRI) with hepatobiliary-specific contrast agents such as gadoxetate enhances detection of liver lesions (45). If size decrease or stable disease is achieved from treatment, combined or staged local parenchymal tumor destruction therapy may be used, depending on the tumor stage $(5,35)$.

Progression of disease can occur in different distinguishable metastatic patterns to aid treatment approach (46). Those who develop new metastases after chemotherapy can be transitioned to the treatment algorithm for surgically untreatable liver-dominant disease (borderline resectable) $(5,35)$. The expert panelists agreed that those patients who have disease that progresses on first-line chemotherapy and experience lesion growth, but who remain surgically 


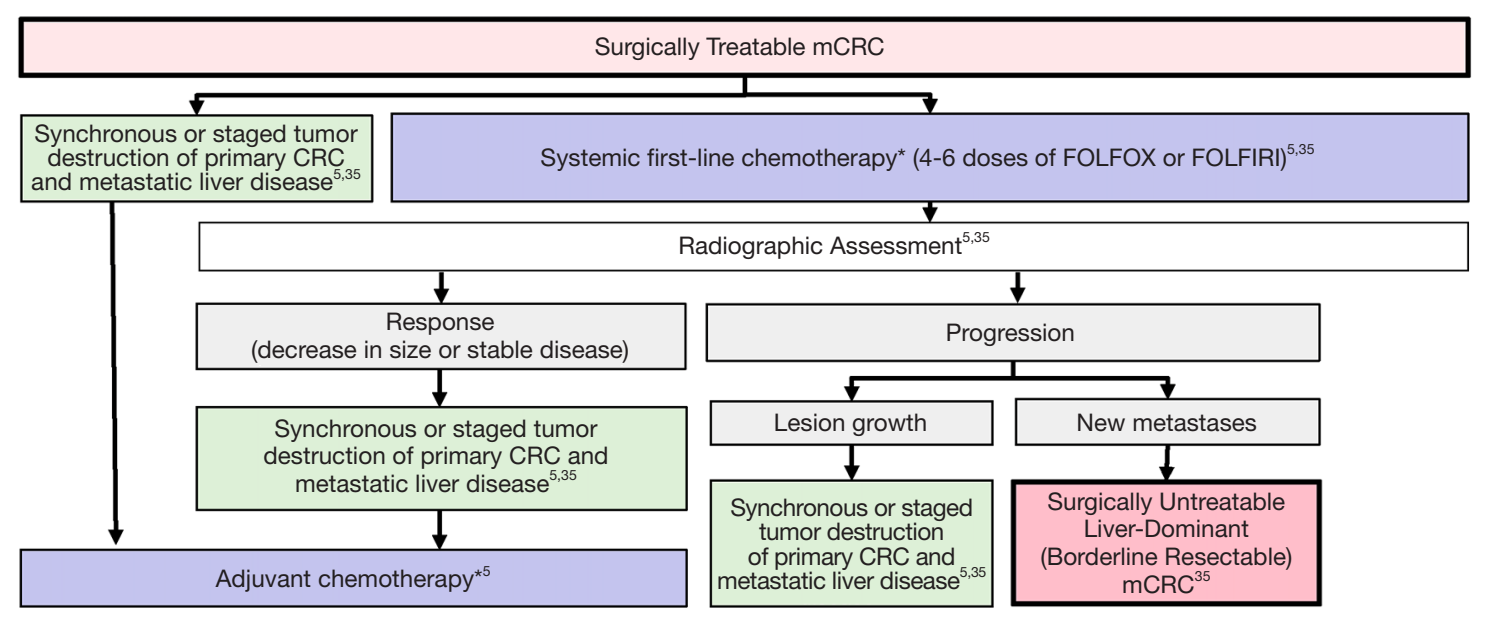

*Consider perioperative chemotherapy and adjuvant chemotherapy in appropriate cases. Selected patients may be appropriate to go straight to surgery.

Supporting evidence

5. National Comprehensive Cancer Network Clinical Practice Guidelines in Oncology. Colon Cancer. Version 4.2018. [NCCN Category

2A Recommendation: Based on lower-level evidence, there is uniform NCCN consensus that the intervention is appropriate]

35. Van Cutsem E, Cervantes A, Adam R, et al. Ann Oncol. 2016;27(8):1386-1422. [ESMO Level IV, grade B recommendation:

Retrospective cohort or case-control studies, strong or moderate evidence for efficacy with limited clinical benefit, generally

recommended]

Figure 5 Treatment algorithm for patients with surgically treatable liver-dominant (resectable) metastatic colorectal cancer (mCRC). ESMO, European Society for Medical Oncology; NCCN, National Comprehensive Cancer Network.

treatable, could be considered for local parenchymal tumor destruction therapy $(5,35)$. Ablation may provide acceptable oncologic outcomes for selected patients with small liver metastases that can be ablated with sufficient margins (5). Clinical studies have shown that 5- and 10-year OS rates for patients who have undergone ablation, for lesions that meet size criteria specifically, are comparable to reported survival rates after surgical resection $(39,40)$. However, this similarity in survival rates is contingent upon the adherence to several factors, including tumor size as well as sufficient experience of the ablating professional $(40,41)$.

While utility of SIRT with Y-90 in surgically treatable liver-dominant mCRC is not well defined, it may have a role in selected patients. For example, for patients with a radiographic complete response to initial chemotherapy of a portion of lesions, SIRT with Y-90 has been described as a time-delaying and chemotherapy-sparing strategy to treat the remaining visible lesions with the goal of extending the time before subsequent chemotherapy is initiated $(47,48)$. The potential for liver damage with chemotherapy emphasizes the importance of limiting the number of treatment cycles (9). SIRT with Y-90 may also be used to expedite treatment of metastases in chemotherapy-naïve patients who cannot receive timely chemotherapy due to inability to tolerate standard agents or limited access to certain healthcare services (32).

Patients who progress on first-line chemotherapy with or without biologics have a worse overall prognosis than those who respond to treatment. This makes some clinicians hesitant to proceed directly to surgical treatment, even in patients with technically surgically treatable disease despite progression. The expert panelists discussed whether SIRT with Y-90 should be considered for use in this subset of patients. SIRT with Y-90 has been used as a complementary therapy in conjunction with second-line chemotherapy in patients with disease that failed to respond to first-line neoadjuvant chemotherapy $(35,49,50)$. The SIRFLOX and FOXFIRE (SF/FFG) studies were conducted to investigate the early use of SIRT with Y-90 in patients with surgically untreatable $\mathrm{mCRC}$ and found that the addition of SIRT with Y-90 to FOLFOX-based first-line chemotherapy in patients with liver-dominant or liver-only mCRC did not improve progression-free survival (PFS), though results may have been biased by the enrollment of patients with significant extrahepatic disease (51). However, treatment with SIRT with Y-90 did significantly delay disease progression in the liver, which suggests that additional research is likely merited (16). In view of these 
first indications of benefit for liver control, there is ample opportunity for additional investigation to strengthen the evidence $(14,51)$. Further study is needed to determine whether this treatment can be clinically adopted, but based on data available, there is insufficient evidence to support a definitive role for the use of SIRT with Y-90 in patients with limited disease at this time (51). Additional clinical trials are required to determine if there is a role for SIRT with Y-90 in CRCLM with limited metastatic disease. (18). The expert panelists recognized that SIRT with Y-90 could be justified as first-line therapy in certain cases for selected patients with liver-dominant disease (16).

\section{Category 2: Surgically untreatable liver-dominant (borderline resectable) mCRC}

Patients in this category have liver-limited unresectable disease that, because of involvement of critical structures, cannot be resected unless regression is accomplished (5). Close proximity of the tumor to normal vital hepatic structures, such as the portal venous bifurcation, bile duct bifurcation, and/or hepatic veins, could potentially disqualify patients from surgery (52). An anticipated positive margin is an unfavorable feature that may make a patient not eligible for up-front resection. While there is no generally accepted definition of what constitutes a positive margin of resection, it has been defined as the presence of exposed tumor along the line of transection or the presence of tumor cells at the line of transection $(<1 \mathrm{~mm})$ detected by histologic examination $(5,44)$. Shrinking the tumor and disengaging it from vital structures can increase the chance of becoming eligible for hepatic resection (52). An adequate future liver remnant (FLR) is imperative to avoid posthepatectomy liver failure and death (52); some patients may lack an adequate FLR and may require an intervention to convert to resectability (5).

The goal of treatment is to convert the patient from a surgically untreatable to resectable status (5). This may often involve selecting a regimen most likely to elicit a rapid treatment response rather than a prolonged chemotherapy course that increases the risk of liver toxicity $(5,9)$.

The treatment of patients with surgically untreatable liver-dominant (borderline resectable) $\mathrm{mCRC}$ is outlined in Figure 6 (5,14,18,28,35,53-63). Preoperative chemotherapy may be used in appropriate cases in an attempt to downsize CRCLM and convert them to a resectable status (5). Patients with a borderline resectable status can be started on systemic chemotherapy, preferably with biologics, as the addition of biologics has been shown to increase treatment response $(5,35)$.

Radiographic assessment must be performed often to assess for extrahepatic metastases and local recurrence $(5,33)$. To limit the development of hepatotoxicity, patients whose disease becomes surgically treatable can be considered to have moved laterally to the algorithm for patients with surgically treatable liver-dominant mCRC, where surgery (e.g., staged tumor destruction) can be considered as soon as possible $(5,35)$.

Patients who respond to treatment but whose liver reserves remain inadequate may undergo intervention to induce contralateral liver hypertrophy and then may be reassessed for surgical treatability $(5,35)$. When the FLR is anticipated to be marginal, there are several options for improving liver reserves and inducing contralateral hypertrophy, to allow for safe major hepatectomy (64). These include portal vein embolization (PVE), SIRT with Y-90, 2-stage hepatectomy, and associating liver partition with portal vein ligation (PVL) for staged hepatectomy (ALPPS) $(57,62,64)$. Among these, SIRT with Y-90 has the advantage of inducing contralateral hypertrophy while also exerting anti-tumoral effects $(24,35,53,65)$. However, it is important to remember that hypertrophy induction after SIRT with Y-90 is slower than after PVE (65). To illustrate the FLR growth curve after treatment with SIRT with Y-90, FLR in one study increased to $23 \%$ within $1-3$ months after treatment, and then increased to $31-34 \%$ and $40-45 \%$ after 6 and 12 months, respectively (65). The anti-tumoral effect in the treated liver segments offered by SIRT with Y-90 allows for a longer interval to surgery and more time to develop contralateral hypertrophy. This prolonged waiting period has some benefits, including the possibility to assess for previously undetected contralateral metastases (65). If FLR hypertrophy is insufficient after SIRT with Y-90, a subsequent PVE/PVL treatment can be considered (65). After contralateral hypertrophy is achieved (4-6 weeks with PVE without interval chemotherapy, more than 6 weeks with chemotherapy, or 3 months after SIRT with Y-90), reassessment of liver regeneration and a secondstage hepatectomy may be performed, possibly followed by additional chemotherapy $(18,59,63,65)$.

If metastatic lesions remain surgically untreatable, patients can be assessed to determine whether they require more chemotherapy or induction of contralateral hypertrophy $(5,35)$. Patients who progress to advanced disease can be treated according to the algorithm for advanced surgically untreatable liver-dominant (unresectable) disease $(5,35)$.

SIRT with Y-90 has been used in patients with surgically untreatable (borderline resectable) mCRC who need a chemotherapy holiday or whose disease has failed available chemotherapeutic options as local disease control to 


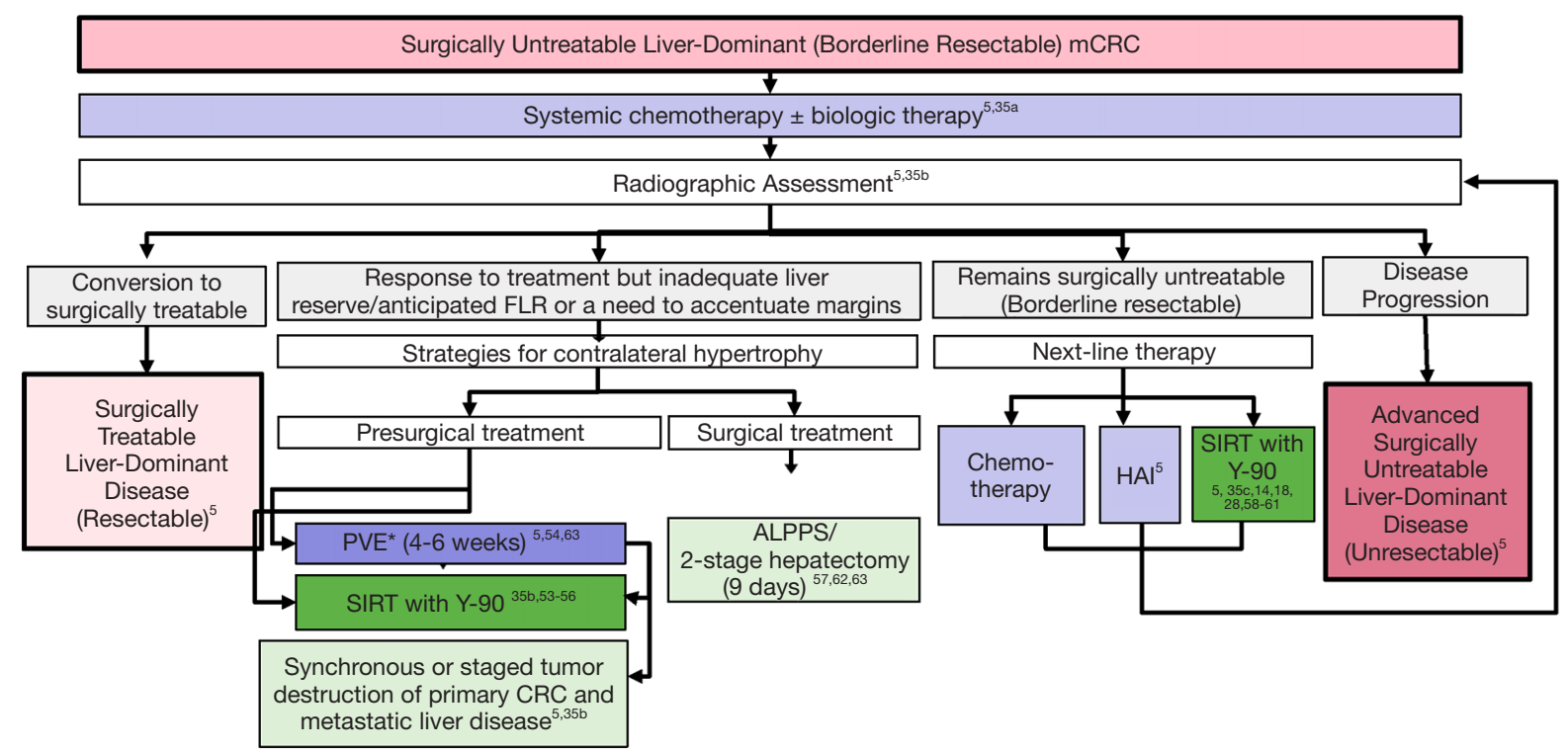

*In selected cases, additional liver-directed therapy is required for further augmentation (eg, transarterial chemoembolization, hepatic vein embolization, etc).

\section{Supporting evidence}

5. National Comprehensive Cancer Network Clinical Practice Guidelines in Oncology. Colon Cancer. Version 4.2018. [NCCN Category 2A Recommendation:

Based on lower-level evidence, there is uniform NCCN consensus that the intervention is appropriate]

14. Garlipp B, Gibbs P, Guy A, et al. J Clin Oncol. 2017;35(15)[suppl]:3532. [Level 2a]

18. Abdalla EK, Bauer TW, Chun YS, et al. HPB (Oxford). 2013;15(2):119-130. [Level 5]

28. Baltatzis M, Siriwardena AK. Dig Surg. 2019;36(4):273-280.. [Level 1a]

35. Van Cutsem E, Cervantes A, Adam R, et al. Ann Oncol. 2016;27(8):1386-1422

a) [ESMO Level II, grade A recommendation: Evidence from RCTs and strong evidence for efficacy with a substantial clinical benefit, strongly

recommended]

b) [ESMO Level IV, grade B recommendation: Retrospective cohort or case-control studies, strong or moderate evidence for efficacy with limited clinical benefit, generally recommended]

c) [ESMO Level II, grade B recommendation: Evidence from RCTs and strong or moderate evidence for efficacy with limited clinical benefit, generally recommended]

53. Pardo F, Sangro B, Lee RC, et al. Ann Surg Oncol. 2017:24(9):2465-2473. [Level 4]

54. Garlipp B, de Baere T, Damm R et al. Hepatology. 2014;59(5):1864-1873. [Level 2b]

55. Shah JL, Zendejas-Ruiz IR, Thornton LM, et al. J Gastrointest Oncol. 2017;8(3):E43-E51. [Level 4]

56. Vouche M, Lewandowski RJ, Atassi R, et al. J Hepatol. 2013;59(5):1029-1036. [Level 4]

57. Vivarelli M, Vincenzi P, Montalti R, et al. PLoS One. 2015;10(12):e0144019. [Level 4- Honorable mention]

58. Moir JAG, Burns J, Barnes J, et al. Br J Surg. 2015;102(12):1533-1540. [Level 4]

59. Hoffmann RT, Jakobs TF, Kubisch CH, et al. Eur J Radiol. 2010;74(1):199-205. [Level 4]

60. Wright GP, Marsh JW, Varma MK, et al. Ann Surg Oncol. 2017;24(4):906-913. [Level 4]

61. Golfieri R, Mosconi C, Giampalma E, et al. Radiol Med. 2015;120(8):767-776. [Level 4]

62. Schnitzbauer AA, Lang SA, Goessmann H, et al. Ann Surg. 2012;255(3):405-414. [Level 4]

63. Schnitzbauer AA. Vis Med. 2017;33(6):435-441.[Level 2a]

Figure 6 Treatment algorithm for patients with surgically untreatable liver-dominant (borderline resectable) metastatic colorectal cancer (mCRC). ALPPS, associating liver partition and portal vein ligation for staged hepatectomy; ESMO, European Society for Medical Oncology; FLR, future liver remnant; HAI, hepatic artery infusion; PVE, portal vein embolization; RCTs, randomized controlled trials; SIRT, selective internal radiation therapy.

treat the dominant metastatic side of the liver, while chemotherapy is stopped, with the goal of improving liver PFS $(5,35,36,48,61)$. As systemic treatment of mCRC results in altered tumor biology and consecutively acquired molecular resistance to therapy, chemotherapy-sparing or time-delaying strategies may be used to allow the disease to reappear for the purpose of further analysis (11). SIRT with Y-90 has been used to fill the gap in treatment as a consolidation therapy in patients after first-line chemotherapy $(47,66)$. Even in patients who show radiographic complete response, it is understood that chemotherapy alone does not result in a pathologic "complete" response in $80 \%$ of patients $(35,48,67)$. SIRT with Y-90 has also been used to obtain additional tumor reduction beyond chemotherapy as an adjunct to second-line or later treatment (14,24,28,48,58-61), as well as for the induction of contralateral hypertrophy in 
patients with inadequate liver reserves $(24,35,48,53-56)$.

In patients with surgically untreatable (borderline resectable) mCRC, SIRT with Y-90 has been used to facilitate downsizing of liver-dominant mCRC tumors to convert selected patients to eligibility for surgical treatment $(14,18)$ and as adjuvant therapy to decrease or stabilize tumor bulk before the patient undergoes systemic chemotherapy, lesion ablation, or liver resection (32).

\section{Category 3: Advanced surgically untreatable liver-dominant (unresectable) mCRC}

Patients with advanced surgically untreatable liverdominant (unresectable) mCRC are not amenable to local parenchymal tumor destruction therapy of CRCLM $(5,35)$. These patients are usually treated with systemic chemotherapy (with or without biologic agents). Many patients eventually become unresponsive to chemotherapy (chemotherapy refractory) or are unable to tolerate multiple cycles of chemotherapy (chemotherapy intolerant) (36).

Patients with advanced mCRC who have progressed after standard first- and second-line therapies have limited treatment options (36). The aim of third-line treatments is to prolong life, improve symptoms, and maintain quality of life; and disease management is often restricted to best supportive care with palliative intent $(6,36)$. The goal of care centers on less-intensive treatments and focuses on disease palliation and monitoring (5,35). Control of liver disease is critical to ameliorating clinical outcomes, and subsequently, the length and quality of life $(16,35,68)$.

The treatment of patients with advanced surgically untreatable liver-dominant (unresectable) $\mathrm{mCRC}$ is outlined in Figure $7(5,14,18,25,28,31,35,61)$. Patients with advanced surgically untreatable liver-dominant disease should be started on systemic chemotherapy with or without biologics $(5,35)$.

Radiographic reassessment must be performed after chemotherapy to assess response $(5,35)$. If response occurs, patients should be reassessed for surgical eligibility; if adequate FLR is achieved for clearance, a staged local parenchymal tumor destruction therapy may be indicated $(5,35)$. If disease remains liver-dominant and surgically untreatable, SIRT with Y-90 followed by systemic chemotherapy or chemotherapy only of the whole liver can be considered $(18,35)$.

The expert panelists agreed that patients should be switched to next-line chemotherapy with or without biologics if disease progresses with standard systemic chemotherapy, with SIRT with Y-90 as an option in cases of chemotherapy resistance $(5,18,35)$. If disease progresses with next-line therapy, SIRT with Y-90 followed by systemic chemotherapy or chemotherapy only of the whole liver is an option $(5,18,35)$. If disease is diffuse, chemotherapy should be continued with or without biologics, maintaining the option for future treatment $(5,35)$. For patients who truly have surgically untreatable liver-dominant disease, and who may never be candidates for local parenchymal tumor destruction therapy, whole-liver treatment with Y-90 is a possible strategy $(5,18,25,35,48)$.

SIRT with Y-90 has been recommended for use as an alternative therapy in the treatment of chemotherapyresistant/-refractory patients with liver-dominant mCRC $(5,36)$. It may be used for local control in patients with liver-dominant disease that remains surgically untreatable after second-line chemotherapy, with the goal of improving liver PFS (i.e., patients who are intolerant to chemotherapy or who need a chemotherapy holiday) $(5,18,31,35,36,48,61)$. Additionally, SIRT with Y-90 may be used as a chemotherapy-sparing or time-delaying strategy in patients with small-volume disease that is no longer detectable after chemotherapy, with the goal of allowing the disease to reappear for the purpose of further analysis (35).

SIRT with Y-90 has been used as salvage therapy for patients with diffuse liver-dominant disease that has failed to respond to multiple treatments $(5,6,18,25,31,61)$. In a phase III RCT, Hendlisz and colleagues provided Level 1 evidence for the survival benefit of SIRT with Y-90 $(8,31)$. In the study, 44 patients heavily pretreated for unresectable liver-limited $\mathrm{mCRC}$ received either infusional 5-FU alone or Y-90 with concomitant infusional 5-FU (31). SIRT with Y-90 significantly prolonged liver tumor growth control (both local and overall disease progression) over protracted intravenous $\mathrm{FU}$ treatments alone and did not increase the toxicity of FU (31). Consideration for SIRT with Y-90 should be given as the patient progresses through the algorithm, with the concept that chemotherapy can be hepatotoxic, and SIRT with Y-90 should be introduced for liver control before the liver has been damaged severely by chemotherapy $(9,35)$.

\section{Discussion}

Through a comprehensive, data-driven modified Delphi consensus model, this multidisciplinary work presents the first described treatment algorithm for CRCLM that incorporates SIRT with Y-90 and provides novel definitions for CRCLM disease categories based on surgical eligibility. The resectable, borderline resectable, and unresectable mCRC disease categories are clearly defined to provide a standardized surgical reference framework for 


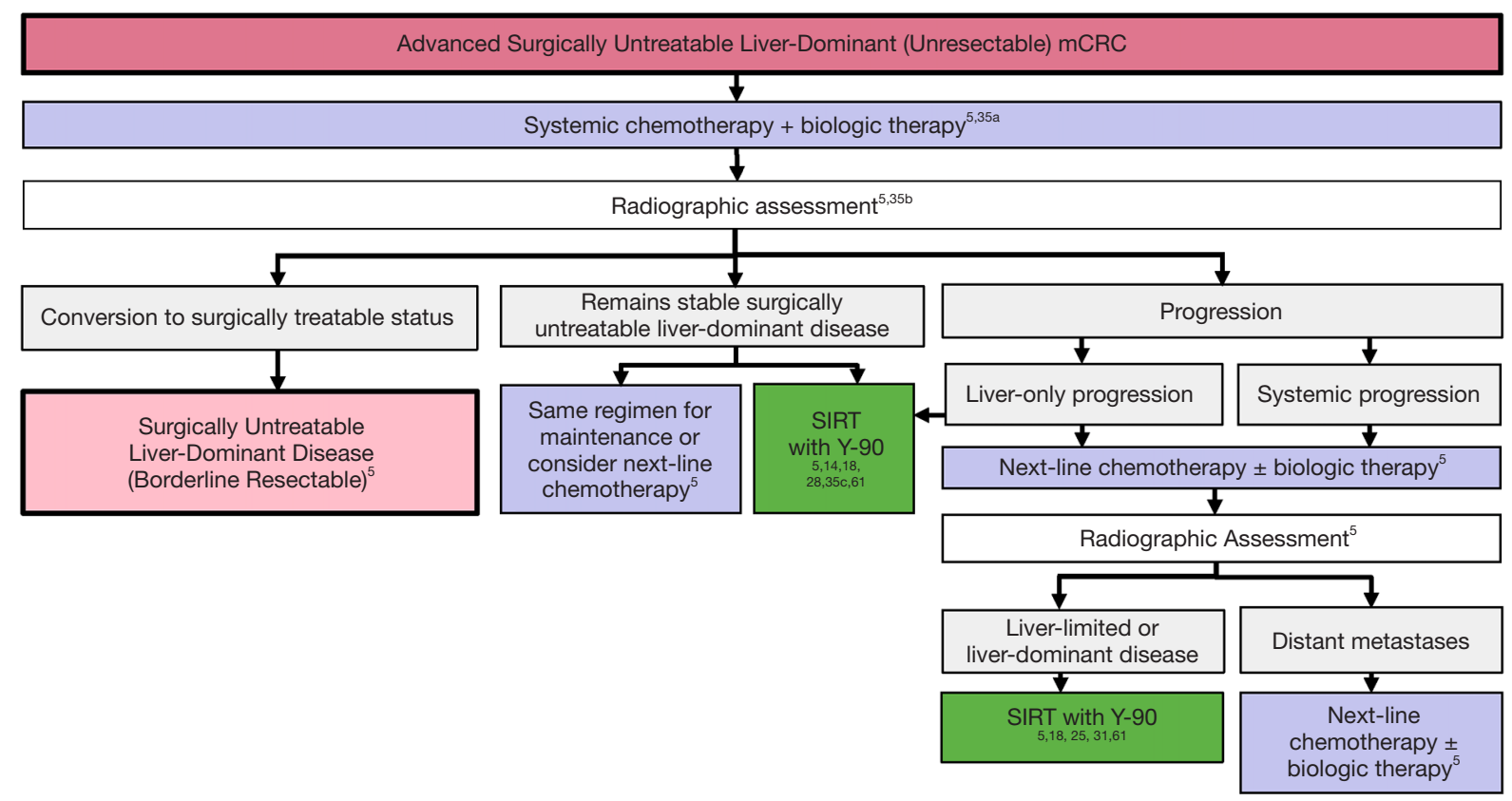

Supporting evidence

5. National Comprehensive Cancer Network Clinical Practice Guidelines in Oncology. Colon Cancer. Version 4.2018. [Category 2A: Based on lower-level evidence, there is uniform NCCN consensus that the intervention is appropriate]

14. Garlipp B, Gibbs P, Guy A, et al. J Clin Oncol. 2017;35(15)[suppl]:3532. [Level 1b]

18. Abdalla EK, Bauer TW, Chun YS, et al. HPB (Oxford). 2013;15(2):119-130. [Level 5]

25. Cosimelli M, Golfieri R, Cagol PP, et al. Br J Cancer. 2010;103(3):324-331. [Level 2b]

28. Baltatzis M, Siriwardena AK. Dig Surg. 2019;36(4):273-80. [Level 1a]

31. Hendlisz A, Van den Eynde M, Peeters M, et al. J Clin Oncol. 2010;28(23):3687-3694. [Level 1b]

35. Van Cutsem E, Cervantes A, Adam R, et al. Ann Oncol. 2016;27(8):1386-1422.

a) [ESMO Level I, grade B recommendation: Evidence from RCTs of good methodological quality or meta-analyses of well-conducted randomised trials without heterogeneity, strong or moderate evidence for efficacy with limited clinical benefit, generally recommended]

b) [ESMO Level IV, grade B recommendation: Retrospective cohort or case-control studies, strong or moderate evidence for efficacy with

limited clinical benefit, generally recommended]

c) [ESMO Level II, grade B recommendation: Evidence from RCTs and strong or moderate evidence for efficacy with limited clinical benefit, generally recommended]

61. Golfieri R, Mosconi C, Giampalma E, et al. Radiol Med. 2015;120(8):767-776. [Level 4]

Figure 7 Treatment algorithm for patients with advanced surgically untreatable liver-dominant (unresectable) metastatic colorectal cancer (mCRC). ALPPS, associating liver partition and portal vein ligation for staged hepatectomy; ESMO, European Society for Medical Oncology; FLR, future liver remnant; HAI, hepatic artery infusion; PVE, portal vein embolization; RCTs, randomized controlled trials; SIRT, selective internal radiation therapy.

mCRC treatment. These definitions are consistent with oncologic definitions for other tumor types, mirroring the classifications used in pancreatic cancer (42). Although several independent groups have attempted to articulate these categories $(34,69)$, none have specifically addressed the diverse roles of SIRT with Y-90 in relation to other treatments. The proposed novel stratification method of surgical treatability establishes specific therapy endpoints to allow physicians to ultimately improve quality of care. Moreover, the presented algorithms allow for entry of patients with various tumor characteristics at different points of treatment with the possibility of moving laterally and vertically as they progress through their treatment pathway. The new term local parenchymal tumor destruction therapy is meant to start a discourse about accommodating treatments like ablation, resection, and future locoregional treatments into the schema of treatment for patients with mCRC.

Though SIRT with Y-90 is currently used as an endof-line treatment modality, it may be effective at multiple points in the algorithm of mCRC management $(32,48,70)$, e.g., to treat small metastases completely (32), as a first-line therapy for hepatic CRCLM alone (35) or in combination with chemotherapy (71-73), in combination with secondor third-line chemotherapy (26), and as salvage therapy for chemotherapy-refractory patients $(25,74,75)$. In addition, 
SIRT with Y-90 can be used as a part of clinical strategies such as control of local liver progression, induction of contralateral hypertrophy, or treatment of the whole liver $(18,35)$. These studies demonstrate that SIRT with Y-90 is able to prolong intrahepatic disease control and improve OS in specific clinical situations (24). The comprehensive treatment algorithm in liver-dominant $\mathrm{mCRC}$ shared here includes opportunities where SIRT with Y-90 may be considered and is supported by available clinical data.

When contemplating therapies, practitioners must first consider a comprehensive multimodal treatment plan while also recognizing temporal factors. For example, contralateral hypertrophy after SIRT with Y-90 develops at a slower pace (3-12 months) than after PVE (6 weeks) $(18,59,65)$. These differences may allow for these 2 procedures to be used to achieve different treatment goals (10), such as immediate resection after hypertrophy (5) or to allow a longer interval to surgery to assess for previously undetected metastases (65).

In addition, selecting appropriate patients suited to SIRT with Y-90 is certainly the key to treatment success $(6,10)$. Patients more likely to benefit from SIRT with Y-90 may include those with no extrahepatic metastases, fewer than 6 tumors, and a tumor-to-liver volume percentage of less than $25 \%(6,76)$. Conversely, patients with extensive tumor volume, more previous lines of chemotherapy, poor radiological response, low preoperative hemoglobin, higher carcinoembryonic antigen and/or cancer antigen 19-9 levels $(30,76)$, KRAS mutations (77), and poor preoperative liver function may show poorer response to treatment $(78,79)$. More investigation regarding these predictors of response is warranted. Appropriate patient selection and coordination of care may require management by a diverse treatment team (including hepatologists, oncologists, HPB surgeons, radiologists, interventional radiologists, radiation oncologists, nuclear medicine physicians, and nurses) $(9,36)$. Especially challenging patients may benefit from review by a dedicated multidisciplinary tumor board, such as the method employed by the REsect study in which patient scans were reviewed for resectability of liver metastases by 3-5 blinded expert surgeons (from a panel of 15). In that study, resectability was deemed when $60 \%$ of reviewers assessed a patient as having resectable disease, which is a way of ensuring treatment consistency (14). Assessment of patients by a multidisciplinary team that includes qualified HPB surgeons may expedite the process of diagnosis and decision-making in the management of patients in different surgical eligibility categories, ultimately optimizing the treatment pathway for each patient $(4,9)$.

Though there is general agreement about the sequencing of chemotherapeutic treatments in first- and subsequentline treatment for mCRC (11), it is not yet clear how to optimally sequence systemic treatments and locoregional therapies (80). Ablation after SIRT with Y-90 in heavily pretreated patients has been shown to be possible. In some patients, SIRT is able to downsize liver metastases to enable subsequent ablation therapy, allowing more patients to have a complete response after minimally invasive therapy (59). How different locoregional treatments and other emerging technologies fit into the overarching schema, as well as how they can be optimally combined and sequenced for treatment of patients with mCRC, is still unclear (80).

Although SIRT with Y-90 may result in improved tumor response rates and OS, treatment must balance the desire for life prolongation and quality of life with the hazards of aggressive treatments, such as the manifestations of longterm hepatotoxicity from Y-90 $(30,81)$. SIRT with Y-90 is generally well tolerated with limited liver toxicity, and most patients have no or mild procedure-related symptoms $(48,82)$. However, if a significant number of radioactive particles reaches non-targeted organs such as the lung, the gastrointestinal tract, or the gallbladder, radiation exposure may lead to future complications (82). Radiation to the liver can produce liver injury and fibrosis secondary to radiation-induced fibrotic tissue remodeling, which results in increased portal pressures and enlargement of the spleen $(81,83)$. Several studies have noted the presence of Y-90-induced liver fibrosis with corresponding changes in hepatic volumes and portal hypertension after treatment $(83,84)$. Furthermore, as SIRT with Y-90 is administered to patients who have typically received systemic anti-cancer therapies as a concurrent or subsequent therapy, drug-drug interactions and toxicities are expected, such as radiation sensitization, or enhancement of expected SIRT with Y-90related adverse events (48). However, although the longterm effects of Y-90 treatment on hepatic function should be recognized, appropriately timed treatment with SIRT with Y-90 may be able to mitigate some of the toxic effects of chemotherapy on the underlying liver parenchyma, potentially minimizing overall liver damage $(9,35)$.

Although it is solidly based on a foundation of published clinical evidence, this work remains a descriptive analysis founded on the opinions and experiences of selected expert panelists and does not represent a uniform consensus. Also, as SIRT that utilizes resin microspheres is currently the only SIRT modality that has an FDA-approved indication 
for treatment of CRCLM, many of the data supporting the algorithm were drawn from studies examining this treatment. Worthy of mention as well are investigations of interest that explore the use of SIRT with glass microspheres in treating CRCLM (85-87), The current algorithm does not stipulate the role of specific chemotherapies at different decision steps, and it is meant to be a more general interdisciplinary map of possible appropriate treatment decisions. While individual steps for treatment at different disease stages are mentioned within established guidelines, this work is the first to put them together in a cohesive, overarching treatment algorithm. Conversations stimulated by these ideas should pave the way for more concrete recommendations by larger organizations in the future.

\section{Conclusions}

SIRT with Y-90 has the potential to play a major role in all phases of liver mCRC treatment. This work addresses the need to establish standard practices for locoregional therapies in the treatment of patients with mCRC and attempts to delineate the distinct roles of SIRT with Y-90 in patients at different stages of disease-not only in the palliative setting. Development of this algorithm was the result of a multidisciplinary effort to establish a reference framework for the treatment of patients with mCRC that incorporates SIRT with Y-90 and introduces treatment algorithms for 3 distinct disease categories stratified by surgical eligibility. This novel stratification method of surgical treatability establishes specific therapy endpoints to allow physicians to ultimately improve quality of care.

\section{Acknowledgments}

The authors thank Ellen Hagopian, MD, Michael House, MD, Christopher Sonnenday, MD, Robert Martin, MD, Patricio Polanco, MD, Hong Jin Kim, MD, Horacio Asbun, MD, Nelson Royall, MD, Cristina Ferrone, MD, Sean Cleary, MD, William Hawkins, MD, and Shimul Shah, MD for their important intellectual contributions to this work. The authors thank Jenny Cai, MSc; Adia Shy, PhD; Chantal Caviness, MD, PhD; Shannon Hach, MD, ELS; and Edward Gloria of Eubio LLC for publication support for journal submission.

Funding: This work was supported by Sirtex Medical, Inc. Editorial assistance was provided by Eubio LLC, funded by Sirtex Medical, Inc.

\section{Footnote}

Conflicts of Interest: All authors have completed the ICMJE uniform disclosure form (available at http:// dx.doi.org/10.21037/jgo.2020.01.09). Authors received an honorarium for Advisory Board attendance that served as the basis for the modified Delphi consensus exercise. No payment was provided for the time spent authoring this manuscript. DR Jeyarajah, NJ Espat, BC Visser, DA Iannitti, Doyle MBM, $\mathrm{J}$ Kim, and T Thambi-Pillai are or have been consultants to Sirtex Medical, Inc. NJ Espat has served as a speaker on behalf of Sirtex. DR Jeyarajah has served as a consultant to Ethicon.

Etbical Statement: The authors are accountable for all aspects of the work in ensuring that questions related to the accuracy or integrity of any part of the work were appropriately investigated and resolved.

Open Access Statement: This is an Open Access article distributed in accordance with the Creative Commons Attribution-NonCommercial-NoDerivs 4.0 International License (CC BY-NC-ND 4.0), which permits the noncommercial replication and distribution of the article with the strict proviso that no changes or edits are made and the original work is properly cited (including links to both the formal publication through the relevant DOI and the license). See: https://creativecommons.org/licenses/by-nc$\mathrm{nd} / 4.0 \%$.

\section{References}

1. Zampino MG, Magni E, Ravenda PS, et al. Treatments for colorectal liver metastases: a new focus on a familiar concept. Crit Rev Oncol Hematol 2016;108:154-63.

2. Hugen $\mathrm{N}$, van de Velde CJ, de Wilt JH, et al. Metastatic pattern in colorectal cancer is strongly influenced by histological subtype. Ann Oncol 2014;25:651-7.

3. Valderrama-Treviño AI, Barrera-Mera B, Ceballos-Villalva JC, et al. Hepatic metastasis from colorectal cancer. Euroasian J Hepatogastroenterol 2017;7:166-75.

4. Weledji EP. Centralization of liver cancer surgery and impact on multidisciplinary teams working on stage IV colorectal cancer. Oncol Rev 2017;11:331.

5. Network NCC. Clinical practice guidelines in oncology. Colon cancer. Version 4. 2018.

6. White J, Carolan-Rees G, Dale M, et al. Analysis of a national programme for selective internal radiation therapy 
for colorectal cancer liver metastases. Clin Oncol (R Coll Radiol) 2019;31:58-66.

7. Folprecht G, Gruenberger T, Bechstein WO, et al. Tumour response and secondary resectability of colorectal liver metastases following neoadjuvant chemotherapy with cetuximab: the CELIM randomised phase 2 trial. Lancet Oncol 2010;11:38-47.

8. Levy J, Zuckerman J, Garfinkle R, et al. Intra-arterial therapies for unresectable and chemorefractory colorectal cancer liver metastases: a systematic review and metaanalysis. HPB (Oxford) 2018;20:905-15.

9. Alberts SR. Update on the optimal management of patients with colorectal liver metastases. Crit Rev Oncol Hematol 2012;84:59-70.

10. Sag AA, Selcukbiricik F, Mandel NM. Evidence-based medical oncology and interventional radiology paradigms for liver-dominant colorectal cancer metastases. World J Gastroenterol 2016;22:3127-49.

11. Modest DP, Pant S, Sartore-Bianchi A. Treatment sequencing in metastatic colorectal cancer. Eur J Cancer 2019; 109:70-83.

12. Folprecht G, Gruenberger T, Bechstein W, et al. Survival of patients with initially unresectable colorectal liver metastases treated with FOLFOX/cetuximab or FOLFIRI/ cetuximab in a multidisciplinary concept (CELIM study). Ann Oncol 2014;25:1018-25.

13. Young AL, Adair R, Culverwell A, et al. Variation in referral practice for patients with colorectal cancer liver metastases. Br J Surg 2013;100:1627-32.

14. Garlipp B, Gibbs P, van Hazel GA, et al. REsect: Blinded assessment of amenability to potentially curative treatment of previously unresectable colorectal cancer liver metastases (CRC LM) after chemotherapy \pm radioembolization (SIRT) in the randomized SIRFLOX trial. J Clin Oncol 2017;35:3532.

15. Winter H, Rassam J, Virdee PS, et al. Hepatic resection following selective internal radiation therapy for colorectal cancer metastases in the FOXFIRE clinical trial: clinical outcomes and distribution of microspheres. Cancers (Basel) 2019;11;1155.

16. van Hazel GA, Heinemann V, Sharma NK, et al. SIRFLOX: randomized phase III trial comparing firstline mFOLFOX6 (plus or minus bevacizumab) versus mFOLFOX6 (plus or minus bevacizumab) plus selective internal radiation therapy in patients with metastatic colorectal cancer. J Clin Oncol 2016;34:1723-31.

17. Jones RP, Poston GJ. Resection of liver metastases in colorectal cancer in the era of expanding systemic therapy.
Annu Rev Med 2017;68:183-96.

18. Abdalla EK, Bauer TW, Chun YS, et al. Locoregional surgical and interventional therapies for advanced colorectal cancer liver metastases: expert consensus statements. HPB (Oxford) 2013;15:119-30.

19. Ackerman NB. The blood supply of experimental liver metastases. IV. Changes in vascularity with increasing tumor growth. Surgery. 1974;75:589-96.

20. Ensminger WD, Rosowsky A, Raso V, et al. A clinicalpharmacological evaluation of hepatic arterial infusions of 5-fluoro-2'-deoxyuridine and 5-fluorouracil. Cancer Res 1978;38:3784-92.

21. Sofocleous CT, Violari EG, Sotirchos VS, et al. Radioembolization as a salvage therapy for heavily pretreated patients with colorectal cancer liver metastases: factors that affect outcomes. Clin Colorectal Cancer 2015;14:296-305.

22. Mahnken AH. Current status of transarterial radioembolization. World J Radiol 2016;8:449-59.

23. Abdel-Rahman O, Cheung WY. Integrating systemic therapies into the multimodality treatment of resectable colorectal liver metastases. Gastroenterol Res Pract 2018;2018:4326082.

24. Justinger C, Gruden J, Kouladouros K, et al. Histopathological changes resulting from selective internal radiotherapy (SIRT). J Surg Oncol 2018;117:1084-91.

25. Cosimelli M, Golfieri R, Cagol PP, et al. Multi-centre phase II clinical trial of yttrium-90 resin microspheres alone in unresectable, chemotherapy refractory colorectal liver metastases. Br J Cancer 2010;103:324-31.

26. Lim L, Gibbs P, Yip D, et al. A prospective evaluation of treatment with selective internal radiation therapy (SIRspheres) in patients with unresectable liver metastases from colorectal cancer previously treated with 5-FU based chemotherapy. BMC Cancer 2005;5:132.

27. Garlipp B, Gibbs P, Van Hazel GA, et al. Secondary technical resectability of colorectal cancer liver metastases after chemotherapy with or without selective internal radiotherapy in the randomized SIRFLOX trial. Br J Surg 2019;106:1837-46.

28. Baltatzis M, Siriwardena AK. Liver resection for colorectal hepatic metastases after systemic chemotherapy and selective internal radiation therapy with yttrium-90 microspheres: a systematic review. Dig Surg 2019;36:273-80.

29. Sharma RA, Van Hazel GA, Morgan B, et al. Radioembolization of liver metastases from colorectal cancer using yttrium-90 microspheres with concomitant systemic oxaliplatin, fluorouracil, and leucovorin 
chemotherapy. J Clin Oncol 2007;25:1099-106.

30. Damm R, Seidensticker R, Ulrich G, et al. Y90

Radioembolization in chemo-refractory metastastic, liver dominant colorectal cancer patients: outcome assessment applying a predictive scoring system. BMC Cancer 2016;16:509.

31. Hendlisz A, Van den Eynde M, Peeters M, et al. Phase III trial comparing protracted intravenous fluorouracil infusion alone or with yttrium-90 resin microspheres radioembolization for liver-limited metastatic colorectal cancer refractory to standard chemotherapy. J Clin Oncol 2010;28:3687-94.

32. Garrean S, Muhs A, Bui JT, et al. Complete eradication of hepatic metastasis from colorectal cancer by yttrium-90 SIRT. World J Gastroenterol 2007;13:3016-9.

33. Chow FC, Chok KS. Colorectal liver metastases: an update on multidisciplinary approach. World J Hepatol 2019;11:150-72.

34. Vera R, González-Flores E, Rubio C, et al. Multidisciplinary management of liver metastases in patients with colorectal cancer: a consensus of SEOM, AEC, SEOR, SERVEI, and SEMNIM. Clin Transl Oncol 2020;22:647-62.

35. Van Cutsem E, Cervantes A, Adam R, et al. ESMO consensus guidelines for the management of patients with metastatic colorectal cancer. Ann Oncol 2016;27:1386-422.

36. Aranda E, Aparicio J, Bilbao JI, et al. Recommendations for SIR-Spheres Y-90 resin microspheres in chemotherapyrefractory/intolerant colorectal liver metastases. Future Oncol 2017;13:2065-82.

37. Grimes M, Wright, G. Delphi Method. Wiley StatsRef: Statistics Reference Online 2016. doi: 10.1002/9781118445112.stat07879.

38. Levels of Evidence (March 2009). Oxford: Oxford Centre for Evidence-based Medicine; 2019. Accessed December 20, 2019. Available online: https://www.cebm.net/2009/06/ oxford-centre-evidence-based-medicine-levels-evidencemarch-2009/

39. Solbiati L, Ahmed M, Cova L, et al. Small liver colorectal metastases treated with percutaneous radiofrequency ablation: local response rate and long-term survival with up to 10-year follow-up. Radiology 2012;265:958-68.

40. Shady W, Petre EN, Gonen M, et al. Percutaneous radiofrequency ablation of colorectal cancer liver metastases: factors affecting outcomes--a 10-year experience at a single center. Radiology 2016;278:601-11.

41. Wang LJ, Zhang ZY, Yan XL, et al. Radiofrequency ablation versus resection for technically resectable colorectal liver metastasis: a propensity score analysis. World J Surg Oncol 2018;16:207.

42. Soloff EV, Zaheer A, Meier J, et al. Staging of pancreatic cancer: resectable, borderline resectable, and unresectable disease. Abdom Radiol (NY) 2018;43:301-13.

43. Salvatore L, Aprile G, Arnoldi E, et al. Management of metastatic colorectal cancer patients: guidelines of the Italian Medical Oncology Association (AIOM). ESMO Open 2017;2:e000147.

44. Pawlik TM, Scoggins CR, Zorzi D, et al. Effect of surgical margin status on survival and site of recurrence after hepatic resection for colorectal metastases. Ann Surg 2005;241:715-22, discussion 722-4.

45. Patel S, Cheek S, Osman H, et al. MRI with gadoxetate disodium for colorectal liver metastasis: is it the new "imaging modality of choice"? J Gastrointest Surg 2014;18:2130-5.

46. Fernández Moro C, Bozóky B, Gerling M. Growth patterns of colorectal cancer liver metastases and their impact on prognosis: a systematic review. BMJ Open Gastroenterol 2018;5:e00217.

47. Cortesi E, Masi, G, Mancini ML, et al. Radioembolization (SIRT) as a consolidation treatment in colorectal liver metastases after first line chemotherapy. J Clin Oncol 2016;34:e15007.

48. Kennedy A, Brown DB, Feilchenfeldt J, et al. Safety of selective internal radiation therapy (SIRT) with yttrium-90 microspheres combined with systemic anticancer agents: expert consensus. J Gastrointest Oncol 2017;8:1079-99.

49. Pini S, Pinto C, Angelelli B, et al. Multimodal sequential approach in colorectal cancer liver metastases: hepatic resection after yttrium-90 selective internal radiation therapy and cetuximab rescue treatment. Tumori 2010;96:157-9.

50. O'Leary C, Greally M, McCaffrey J, et al. Singleinstitution experience with selective internal radiation therapy (SIRT) for the treatment of unresectable colorectal liver metastases. Ir J Med Sci 2019;188:43-53.

51. Wasan HS, Gibbs P, Sharma NK, et al. First-line selective internal radiotherapy plus chemotherapy versus chemotherapy alone in patients with liver metastases from colorectal cancer (FOXFIRE, SIRFLOX, and FOXFIREGlobal): a combined analysis of three multicentre, randomised, phase 3 trials. Lancet Oncol 2017;18:1159-71.

52. Cucchetti A, Cappelli A, Ercolani G, et al. Selective internal radiation therapy (SIRT) as conversion therapy for unresectable primary liver malignancies. Liver Cancer 2016;5:303-11. 
53. Pardo F, Sangro B, Lee RC, et al. The Post-SIR-Spheres Surgery Study (P4S): Retrospective analysis of safety following hepatic resection or transplantation in patients previously treated with selective internal radiation therapy with yttrium-90 resin microspheres. Ann Surg Oncol 2017;24:2465-73.

54. Garlipp B, de Baere T, Damm R, et al. Left-liver hypertrophy after therapeutic right-liver radioembolization is substantial but less than after portal vein embolization. Hepatology 2014;59:1864-73.

55. Shah JL, Zendejas-Ruiz IR, Thornton LM, et al. Neoadjuvant transarterial radiation lobectomy for colorectal hepatic metastases: a small cohort analysis on safety, efficacy, and radiopathologic correlation. J Gastrointest Oncol 2017;8:E43-51.

56. Vouche M, Lewandowski RJ, Atassi R, et al. Radiation lobectomy: time-dependent analysis of future liver remnant volume in unresectable liver cancer as a bridge to resection. J Hepatol 2013;59:1029-36.

57. Vivarelli M, Vincenzi P, Montalti R, et al. ALPPS procedure for extended liver resections: a single centre experience and a systematic review. PLoS One 2015;10:e0144019.

58. Moir JA, Burns J, Barnes J, et al. Selective internal radiation therapy for liver malignancies. Br J Surg 2015;102:1533-40.

59. Hoffmann RT, Jakobs TF, Kubisch CH, et al. Radiofrequency ablation after selective internal radiation therapy with yttrium90 microspheres in metastatic liver disease-is it feasible? Eur J Radiol 2010;74:199-205.

60. Wright GP, Marsh JW, Varma MK, et al. Liver resection after selective internal radiation therapy with yttrium-90 is safe and feasible: a bi-institutional analysis. Ann Surg Oncol 2017;24:906-13.

61. Golfieri R, Mosconi C, Giampalma E, et al. Selective transarterial radioembolisation of unresectable liverdominant colorectal cancer refractory to chemotherapy. Radiol Med 2015;120:767-76.

62. Schnitzbauer AA, Lang SA, Goessmann H, et al. Right portal vein ligation combined with in situ splitting induces rapid left lateral liver lobe hypertrophy enabling 2-staged extended right hepatic resection in small-for-size settings. Ann Surg 2012;255:405-14.

63. Schnitzbauer AA. A comparison of pitfalls after ALPPS stage 1 or portal vein embolization in small-for-size setting hepatectomies. Visc Med 2017;33:435-41.

64. Clark ME, Smith RR. Liver-directed therapies in metastatic colorectal cancer. J Gastrointest Oncol
2014;5:374-87.

65. Braat MN, Samim M, van den Bosch MA, Lam MG. The role of (90)Y-radioembolization in downstaging primary and secondary hepatic malignancies: a systematic review. Clin Transl Imaging 2016;4:283-95.

66. Cortesi E, Masi G, Mancini M, et al. Radioembolization (SIRT) as a consolidation treatment in colorectal liver metastases after first line chemotherapy: efficacy safety. P-158. Ann Oncol 2016;27:ii47.

67. Benoist S, Brouquet A, Penna C, Julie C, et al. Complete response of colorectal liver metastases after chemotherapy: does it mean cure? J Clin Oncol 2006;24:3939-45.

68. Li L, Yeo W. Value of quality of life analysis in liver cancer: A clinician's perspective. World J Hepatol 2017;9:867-83.

69. Cetin B, Bilgetekin I, Cengiz M, et al. Managing synchronous liver metastases in colorectal cancer. Indian J Surg Oncol 2018;9:461-71.

70. Braat AJ, Huijbregts JE, Molenaar IQ, et al. Hepatic radioembolization as a bridge to liver surgery. Front Oncol 2014;4:199.

71. Gray B, Van Hazel G, Hope M, et al. Randomised trial of SIR-Spheres plus chemotherapy vs. chemotherapy alone for treating patients with liver metastases from primary large bowel cancer. Ann Oncol 2001;12:1711-20.

72. Kosmider S, Tan TH, Yip D, et al. Radioembolization in combination with systemic chemotherapy as first-line therapy for liver metastases from colorectal cancer. J Vasc Interv Radiol 2011;22:780-6.

73. Sharma N, van Hazel G, Findlay M, et al. Using data from the SIRFLOX study to evaluate depth of response within a volumetric model in patients with metastatic colorectal cancer. J Vasc Interv Radiol 2017;28:S160-1.

74. Bester L, Meteling B, Pocock N, et al. Radioembolisation with yttrium-90 microspheres: an effective treatment modality for unresectable liver metastases. J Med Imaging Radiat Oncol 2013;57:72-80.

75. Kennedy AS, Ball D, Cohen SJ, et al. Multicenter evaluation of the safety and efficacy of radioembolization in patients with unresectable colorectal liver metastases selected as candidates for $(90) \mathrm{Y}$ resin microspheres. J Gastrointest Oncol 2015;6:134-42.

76. Kurilova I, Beets-Tan RGH, Flynn J, et al. Factors affecting oncologic outcomes of $90 \mathrm{Y}$ radioembolization of heavily pre-treated patients with colon cancer liver metastases. Clin Colorectal Cancer 2019;18:8-18.

77. Lahti SJ, Xing M, Zhang D, et al. KRAS status as an independent prognostic factor for survival after yttrium-90 radioembolization therapy for unresectable colorectal cancer 
liver metastases. J Vasc Interv Radiol 2015;26:1102-11.

78. Saxena A, Meteling B, Kapoor J, et al. Is yttrium-90 radioembolization a viable treatment option for unresectable, chemorefractory colorectal cancer liver metastases? A large single-center experience of 302 patients. Ann Surg Oncol 2015;22:794-802.

79. Kennedy A, Cohn M, Coldwell DM, et al. Updated survival outcomes and analysis of long-term survivors from the MORE study on safety and efficacy of radioembolization in patients with unresectable colorectal cancer liver metastases. J Gastrointest Oncol 2017;8:614-24.

80. Memon K, Kulik L, Lewandowski RJ, et al. Radioembolization for hepatocellular carcinoma with portal vein thrombosis: impact of liver function on systemic treatment options at disease progression. J Hepatol 2013;58:73-80.

81. Su YK, Mackey RV, Riaz A, et al. Long-term hepatotoxicity of yttrium-90 radioembolization as treatment of metastatic neuroendocrine tumor to the liver. J Vasc Interv Radiol 2017;28:1520-6.

82. Sangro B, Martínez-Urbistondo D, Bester L, et al. Prevention and treatment of complications of selective internal radiation therapy: Expert guidance and systematic review. Hepatology 2017;66:969-82.

83. Jakobs TF, Saleem S, Atassi B, et al. Fibrosis, portal hypertension, and hepatic volume changes induced by intra-arterial radiotherapy with $90 y$ ttrium microspheres. Dig Dis Sci 2008;53:2556-63.

84. Maker AV, August C, Maker VK, et al. Hepatectomy after yttrium-90 (Y90) radioembolization-induced liver fibrosis. J Gastrointest Surg 2016;20:869-70.

85. Abbott AM, Kim R, Hoffe SE, et al. Outcomes of Therasphere radioembolization for colorectal metastases. Clin Colorectal Cancer 2015;14:146-53.

86. Hickey R, Lewandowski RJ, Prudhomme T, et al. 90Y radioembolization of colorectal hepatic metastases using glass microspheres: safety and survival outcomes from a 531-patient multicenter study. J Nucl Med 2016;57:665-71.

87. Lewandowski RJ, Thurston KG, Goin JE, et al. 90Y microsphere (TheraSphere) treatment for unresectable colorectal cancer metastases of the liver: response to treatment at targeted doses of 135-150 Gy as measured by [18F]fluorodeoxyglucose positron emission tomography and computed tomographic imaging. J Vasc Interv Radiol 2005;16:1641-51.
Cite this article as: Jeyarajah DR, Doyle MBM, Espat NJ, Hansen PD, Iannitti DA, Kim J, Thambi-Pillai T, Visser BC. Role of yttrium-90 selective internal radiation therapy in the treatment of liver-dominant metastatic colorectal cancer: an evidence-based expert consensus algorithm. J Gastrointest Oncol 2020;11(2):443-460. doi: 10.21037/jgo.2020.01.09 